\title{
Debye temperatures of uranium chalcogenides from their lattice dynamics
}

\author{
S DURAI* and P BABU \\ Postgraduate and Research Department of Physics, A.M. Jain College, Chennai 600 114, India
}

MS received 13 June 2005; revised 23 September 2005

\begin{abstract}
Phonon dispersion relations in uranium chalcogenides have been investigated using a modified three-body force shell model. From the phonon frequencies, their Debye temperatures are evaluated. Further, on the basis of the spin fluctuation in the heavy fermion uranium compounds, $\mathrm{UPt}_{3}$ and $\mathrm{UBe}_{13}$, the possible superconducting transition temperatures of chalcogenides are theoretically predicted. The $T_{c}$ values are in the same range as of those in $\mathrm{UPt}_{3}$ and $\mathrm{UBe}_{13}$.
\end{abstract}

Keywords. Uranium chalcogenides; $p$-wave electronic superconductor; phonon frequency; Debye temperature; spin fluctuation.

\section{Introduction}

Uranium compounds, besides their important use in reactor technology, possess more interesting and also controversial properties. Hence, over nearly last two decades they have attracted the attention of both experimental and theoretical solid state physicists. Chalcogenides (UX, $X=S, S e, T e)$ show ferromagnetic ordering while the pnictides (UY, Y $=\mathrm{N}, \mathrm{P}, \mathrm{As}, \mathrm{Sb}, \mathrm{Bi}$ ) order antiferromagnetically. Their lattice vibrational properties have been extensively studied. At the same time, $\mathrm{UPt}_{3}$ and $\mathrm{UBe}_{13}$ have gained significance in another way. They belong to the heavy fermion system. They are characterized by large magnetic fluctuations and high values of $\gamma$ which is the coefficient of linear term in specific heat. $\mathrm{UBe}_{13}$ is the first $p$-wave electronic superconductor $\left(T_{\mathrm{c}}=0.905 \mathrm{~K}\right)$ and the next is $\mathrm{UPt}_{3}\left(T_{\mathrm{c}}=0.54 \mathrm{~K}\right)$ (Ott et al 1984; Aeppli et al 1985). The two compounds are characterized by very low coupling coefficient, $\lambda$, the components of which are $\lambda_{\text {ep }}$ (electron-phonon) and $\lambda_{\text {ee }}$ (electron-electron) and very small value of coulomb pseudo potential, $\mu^{*} \sim 0.25$, indicating a weak coupling.

This paper is presented with the main aim to study the lattice vibrations of uranium chalcogenides using the refined three-body force shell model and to obtain the Debye temperature from the phonon frequencies. Using modified McMillan equation and making use of conventional superconductivity theory to predict the possibility of these uranium compounds becoming superconductors on parallel lines with the behaviour of $\mathrm{UPt}_{3}$ and $\mathrm{UBe}_{13}$.

\footnotetext{
*Author for correspondence (bagee29@rediffmail.com)
}

\section{Theoretical background}

\subsection{Evolution of various models in the study of lattice dynamics}

The lattice dynamics of $\mathrm{NaCl}$ was extensively investigated by Kellermann (1940) and the treatment is applicable to the ionic crystals of similar structure. The supporting theories of shell model (Dick and Overhauser 1958), subsequent modifications (Woods et al 1960) and inclusion of three-body forces between ions by Verma and Singh (1969) were the various stages of progress. The discrepancy in three-body force shell model was removed by Verma and Agarwal (1973). The various interactions between ions were taken to be equal in earlier treatment and it was Mohan et al (1986) who applied the three-body force shell model to the lattice dynamics of calcium oxide without making them numerically equal. The results were satisfactory and on that basis lattice dynamics of strontium oxide and cesium fluoride were studied (Durai et al 1987; Mohan and Durai 1988).

In the present work, we have chosen chalcogenides which crystallize in the rock-salt structure and exhibit unusual magnetic and elastic properties.

The earlier works related to such calculations are (i) rigid-ion and shell model by Jackman et al (1986) and (ii) inclusion of long range many-body forces and breathing shell model by Jha and Sanyal (1992). We have applied the modified three-body force shell model to obtain the phonon frequencies of UX $(X=S, S e, T e)$ for the following specific reason. Though the uranium compounds have rock-salt structure, they are more metallic in nature rather than ionic and the curiosity is to know how the theory works in this case. Further, from the frequencies obtained, the Debye temperature, $\theta_{\mathrm{D}}$, is calculated. 


\subsection{Chalcogenides and heavy fermion superconductors}

The uranium compounds, $\mathrm{UPt}_{3}$ and $\mathrm{UBe}_{13}$, are heavy fermion superconductors. Oguchi and Freeman (1985) attempted to account for their superconductivity on the basis of conventional theory. We, inspired by this clue, are of the opinion that the other UX compounds could also attain superconductivity in the range close to that of $\mathrm{UPt}_{3}$ and $\mathrm{UBe}_{13}$. Making use of $\lambda$ and $\mu^{*}$ values similar to those of heavy fermion system, the $T_{\mathrm{c}}$ values have been calculated with the help of modified McMillan (1968) equation for which $\theta_{\mathrm{D}}$ values obtained from the preceding section are useful.

\section{Formulae and calculation}

\subsection{Evaluation of phonon frequencies and Debye} temperature

The three-body force shell model equations in the matrix form are given by

$$
\begin{gathered}
-M \omega^{2} u=\left(R+Z C^{\prime} Z\right) u+\left(T+Z C^{\prime} Y\right) w, \\
0=\left(Y C^{\prime} Z+T\right) u+\left(Y C^{\prime} Y+S\right) w,
\end{gathered}
$$

Table 1. Input parameters.

\begin{tabular}{llllllll}
\hline Compound & $C_{11}$ & $C_{12}$ & $C_{44}$ & $\omega_{\mathrm{LO}}$ & $a_{0}$ & $\alpha_{1}$ & $\alpha_{2}$ \\
\hline US & 3.056 & 0.205 & 0.1702 & 9.50 & 5.489 & 0.40 & 9.875 \\
USe & 2.08 & 0.07 & 0.17 & 6.25 & 5.744 & 0.3 & 11.31 \\
UTe & 1.47 & -0.16 & 0.119 & 4.75 & 6.155 & 0.20 & 13.923
\end{tabular}

$\left(C_{11}, C_{12}, C_{44}\right.$ in $10^{12}$ dynes $/ \mathrm{cm}^{2}, \omega_{\mathrm{LO}}$ in THz, $a_{0}$ in $\AA$ and $\alpha_{1}, \alpha_{2}$ in $\AA^{3}$ (Jha and Sanyal 1992).

Table 2. Model parameters.

\begin{tabular}{lcccccc}
\hline Compound & $Z[Z+12 f(a)]$ & $Z[a(\mathrm{~d} f / \mathrm{d} a)]$ & $A$ & $B$ & $Y_{1}$ & \multicolumn{1}{c}{$Y_{2}$} \\
\hline US & 26.824 & -0.537 & 623.769 & 31.250 & 0.556 & 0.002 \\
USe & 32.130 & -3.376 & 588.832 & 37.432 & 0.497 & 0.128 \\
UTe & 21.462 & 7.481 & 406.298 & -29.128 & 0.608 & 0.205 \\
\hline
\end{tabular}

Table 3. Calculated phonon frequencies $\left(10^{12} \mathrm{~Hz}\right)$ for US.

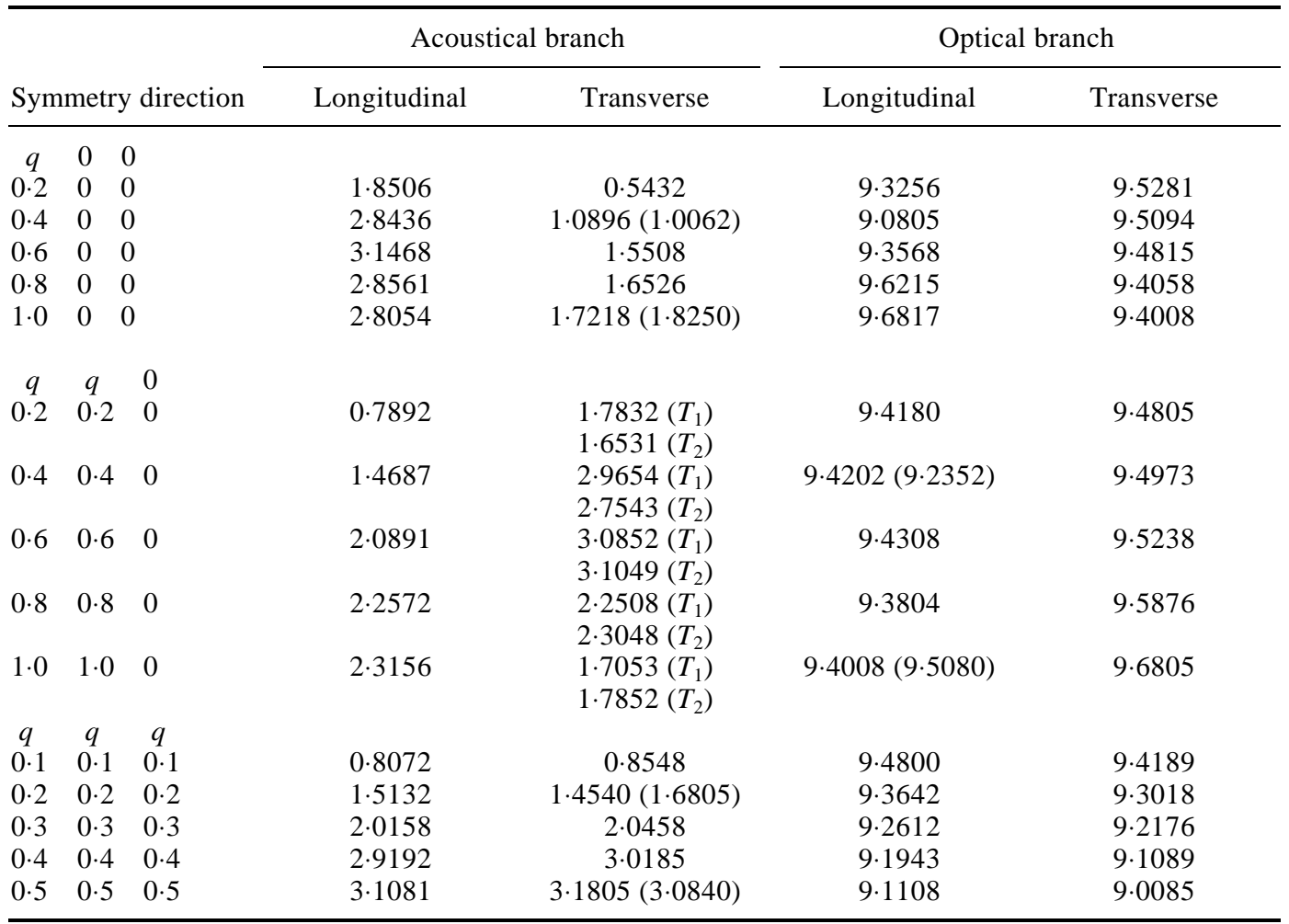

The values in parantheses are from Jackman et al (1986). 
with $Z C^{\prime} Z=Z[Z+12 f(a)]+V$, where $R, S, T$ are matrices specifying core-core, shell-shell and core-shell interactions, respectively and $f(a)$ is related to overlap integrals of electron wave functions. $V$ is the force constant derived from the purely three-body part of the lattice potential. The basic equations pertaining to TSM are the same as given by Agarwal (1977). In the present approach, $R$, $S, T$ elements are treated as different i.e. $R \neq S \neq T$. The $R$ and $C$ elements are calculated from the dimensionless coefficients of Kellermann. Short range force constants $A_{1}, B_{1}, A_{11}$ and $B_{11}$, are used to find out the $T$ matrix elements. Further, the variation in the $T$-matrix elements with respect to symmetry directions are identical to the corresponding variations in the $R$-elements. A simple manipulation leads to the result that $S=2 T-R$. The $V$-matrix elements are calculated using the coefficients (revised values) given by Verma and Singh (1969).

The input data necessary for the calculations of the model parameters are the elastic constants, $C_{11}, C_{12}$, and $C_{44}$, long wave optical frequencies, $w_{\mathrm{LO}}$, and $w_{\mathrm{TO}}$, the lattice constant, $a_{0}$, and the electrical polarizabilities, $\alpha_{1}$ and $\alpha_{2}$. The repulsive forces between ions are connected with polarization of ions and hence the polarizabilities of positive and negative ions, $\alpha_{1}$ and $\alpha_{2}$, are essential for solving the equations. The model parameters are the constants $A, B$, overlap integral constant, $f(a),(a \mathrm{~d} f / \mathrm{d} a)$ and the shell charges, $Y_{1}$ and $Y_{2}$. With these data the secular equation is solved to obtain the phonon frequencies. Using the maximum values of phonon frequency, the Debye temperature, $\theta_{\mathrm{D}}$, is calculated.

\subsection{Estimation of superconducting transition temperatures}

On the basis of strong coupling theory, McMillan (1968) suggested the following equation for the theoretical estimation of superconducting transition temperature, $T_{\mathrm{c}}$,

$$
T_{\mathrm{c}}=\left(\theta_{\mathrm{D}} / 1 \cdot 45\right) \exp \left[-1 \cdot 04(1+\lambda) /\left(\lambda-\mu^{*}(1+0.62 \lambda)\right)\right],
$$

where $\lambda$ is the electron-phonon coupling constant $\left(\lambda_{\mathrm{ep}}\right)$ and $\mu^{*}$ the coulomb coupling constant. However, another factor relevant to the present work is the spin fluctuations. If its influence is to be incorporated in the above equation, then $\lambda$ is to be modified as $\lambda=\lambda_{\text {ep }} /\left(1+\lambda_{\mathrm{sf}}\right)$, where $\lambda_{\mathrm{sf}}$ is the component due to spin fluctuation. $\lambda_{\mathrm{sf}}$ is estimated from a knowledge of mass enhance factor. Since the coupling is not a strong one, there is no chance of large values of $\lambda$ or $\mu^{*}$ being associated with the compounds. In comparison with heavy fermion superconductors, two values have been considered for $\mu^{*}$ and $\lambda_{\text {ep }}$ and $\lambda_{\text {sf }}$ values are chosen keeping in mind the trend of $\theta_{D}$ values. The chosen value for $\lambda$ is around 0.33 and for $\mu^{*}$ the values considered are $0 \cdot 1$ and $0 \cdot 12$. The reason for such selec-

Table 4. Calculated phonon frequencies $\left(10^{12} \mathrm{~Hz}\right)$ for USe.

\begin{tabular}{|c|c|c|c|c|c|c|}
\hline & & & \multicolumn{2}{|c|}{ Acoustical branch } & \multicolumn{2}{|c|}{ Optical branch } \\
\hline \multicolumn{3}{|c|}{ Symmetry direction } & Longitudinal & Transverse & Longitudinal & Transverse \\
\hline$q$ & $0 \quad 0$ & & & & & \\
\hline $0 \cdot 2$ & 00 & & 1.7539 & 0.5178 & $6 \cdot 0028$ & $6 \cdot 2829$ \\
\hline 0.4 & 00 & & 2.6538 & $0.8039(0.9030)$ & $5 \cdot 7096$ & $6 \cdot 2986$ \\
\hline 0.6 & $0 \quad 0$ & & 2.7089 & $1 \cdot 2041$ & $5 \cdot 7008$ & $6 \cdot 3089$ \\
\hline $0 \cdot 8$ & $\begin{array}{ll}0 & 0\end{array}$ & & $2 \cdot 2123$ & 1.4536 & $6 \cdot 0890$ & $6 \cdot 3525$ \\
\hline $1 \cdot 0$ & $\begin{array}{lll}0 & 0\end{array}$ & & 1.8090 & $1.4098(1.5078)$ & $6 \cdot 2428$ & $6 \cdot 3896$ \\
\hline$q$ & $q$ & 0 & & & & \\
\hline $0 \cdot 2$ & $0 \cdot 2$ & 0 & 0.7435 & $\begin{array}{l}1 \cdot 7081\left(T_{1}\right) \\
1 \cdot 8132\left(T_{2}\right)\end{array}$ & $6 \cdot 0972$ & $6 \cdot 1972$ \\
\hline $0 \cdot 4$ & 0.4 & 0 & $1 \cdot 1817$ & $\begin{array}{l}3 \cdot 0817\left(T_{1}\right) \\
3 \cdot 2006\left(T_{2}\right)\end{array}$ & $5 \cdot 5036(5 \cdot 6840)$ & $6 \cdot 2085$ \\
\hline $0 \cdot 6$ & $0 \cdot 6$ & 0 & 1.4813 & $\begin{array}{l}2 \cdot 7090\left(T_{1}\right) \\
2 \cdot 8132\left(T_{2}\right)\end{array}$ & $5 \cdot 4732$ & $6 \cdot 2196$ \\
\hline $0 \cdot 8$ & $0 \cdot 8$ & 0 & 1.6581 & $\begin{array}{l}2 \cdot 0581\left(T_{1}\right) \\
2 \cdot 1054\left(T_{2}\right)\end{array}$ & $5 \cdot 8142$ & $6 \cdot 2253$ \\
\hline $1 \cdot 0$ & $1 \cdot 0$ & 0 & $1 \cdot 8156$ & $\begin{array}{l}1.4108\left(T_{1}\right) \\
1.8132\left(T_{2}\right)\end{array}$ & $6 \cdot 2013(6 \cdot 2240)$ & $6 \cdot 2483$ \\
\hline $\begin{array}{c}q \\
0 \cdot 1\end{array}$ & $q$ & $q$ & & & & \\
\hline $0 \cdot 1$ & $0 \cdot 1$ & $0 \cdot 1$ & $0 \cdot 7431$ & 0.7381 & $6 \cdot 0315$ & $6 \cdot 108$ \\
\hline $0 \cdot 2$ & $0 \cdot 2$ & $0 \cdot 2$ & $1.2492(1.4320)$ & $1 \cdot 2508$ & 5.9518 & 5.9450 \\
\hline $0 \cdot 3$ & $0 \cdot 3$ & $0 \cdot 3$ & 1.7645 & 1.7895 & $5 \cdot 8160$ & $5 \cdot 7480$ \\
\hline $0 \cdot 4$ & 0.4 & $0 \cdot 4$ & $2 \cdot 2108$ & $2 \cdot 3945$ & $5 \cdot 8009$ & $5 \cdot 7021$ \\
\hline $0 \cdot 5$ & $0 \cdot 5$ & $0 \cdot 5$ & $2.7563(2.9084)$ & $2 \cdot 9813$ & $5 \cdot 7843$ & $5 \cdot 6805$ \\
\hline
\end{tabular}

The values in parantheses are from Jackman et al (1986). 
Table 5. Calculated phonon frequencies $\left(10^{12} \mathrm{~Hz}\right)$ for UTe.

\begin{tabular}{|c|c|c|c|c|c|c|}
\hline \multirow{2}{*}{\multicolumn{3}{|c|}{ Symmetry direction }} & \multicolumn{2}{|c|}{ Acoustical branch } & \multicolumn{2}{|c|}{ Optical branch } \\
\hline & & & \multirow[t]{2}{*}{ Longitudinal } & \multirow[t]{2}{*}{ Transverse } & \multirow[t]{2}{*}{ Longitudinal } & \multirow[t]{2}{*}{ Transverse } \\
\hline$q$ & 0 & & & & & \\
\hline $0 \cdot 2$ & 0 & & $1 \cdot 2054$ & 0.2508 & 4.7582 & $4 \cdot 7620$ \\
\hline $0 \cdot 4$ & 0 & & $2 \cdot 1087$ & $0.6574(0.7056)$ & $4 \cdot 2358$ & 4.7519 \\
\hline $0 \cdot 6$ & 0 & & $2 \cdot 2576$ & 0.8508 & 4.0078 & 4.7328 \\
\hline $0 \cdot 8$ & 0 & & 1.5786 & 0.9048 & $4 \cdot 3152$ & 4.7192 \\
\hline $1 \cdot 0$ & 0 & & $1 \cdot 2583$ & $0 \cdot 9048(0 \cdot 8560)$ & $4 \cdot 6829$ & 4.7052 \\
\hline$q$ & $q$ & $q$ & & & & \\
\hline $0 \cdot 2$ & $0 \cdot 2$ & 0 & $1 \cdot 2538$ & $\begin{array}{l}0.3095\left(T_{1}\right) \\
0 \cdot 3783\left(T_{1}\right)\end{array}$ & $4 \cdot 6804$ & 4.7126 \\
\hline $0 \cdot 4$ & $0 \cdot 4$ & 0 & $1 \cdot 8170$ & $\begin{array}{l}0.8172\left(T_{1}\right) \\
1.0056\left(T_{2}\right)\end{array}$ & $4 \cdot 2154(4 \cdot 1582)$ & $4 \cdot 7234$ \\
\hline $0 \cdot 6$ & $0 \cdot 6$ & 0 & $1 \cdot 8090$ & $\begin{array}{l}1 \cdot 2105\left(T_{1}\right) \\
1 \cdot 3248\left(T_{12}\right)\end{array}$ & $4 \cdot 0058$ & $4 \cdot 7325$ \\
\hline $0 \cdot 8$ & $0 \cdot 8$ & 0 & $1 \cdot 3421$ & $\begin{array}{l}1.2359\left(T_{1}\right) \\
1.2864\left(T_{12)}\right)\end{array}$ & $4 \cdot 2814$ & $4 \cdot 7681$ \\
\hline $1 \cdot 0$ & $1 \cdot 0$ & 0 & 0.9050 & $\begin{array}{l}1 \cdot 0897\left(T_{1}\right) \\
1 \cdot 1720\left(T_{12}\right)\end{array}$ & $4.7238(4.9560)$ & $4 \cdot 8091$ \\
\hline$q$ & $q$ & $q$ & & & & \\
\hline $0 \cdot 1$ & $0 \cdot 1$ & $0 \cdot 1$ & $0 \cdot 5198$ & 0.5208 & $4 \cdot 7512$ & $4 \cdot 7678$ \\
\hline $0 \cdot 2$ & $0 \cdot 2$ & $0 \cdot 2$ & $1 \cdot 2108(1.2268)$ & $1 \cdot 3024$ & 4.6278 & $4 \cdot 6013$ \\
\hline $0 \cdot 3$ & $0 \cdot 3$ & $0 \cdot 3$ & $1 \cdot 5321$ & $1 \cdot 7532$ & $4 \cdot 5832$ & $4 \cdot 5032$ \\
\hline $0 \cdot 4$ & $0 \cdot 4$ & $0 \cdot 4$ & 1.7835 & $2 \cdot 0871$ & 4.4972 & $4 \cdot 2081$ \\
\hline $0 \cdot 5$ & $0 \cdot 5$ & $0 \cdot 5$ & $2 \cdot 0028(2 \cdot 1008)$ & $2 \cdot 4092$ & 4.4278 & 4.0075 \\
\hline
\end{tabular}

The values in parantheses are from Jackman et al (1986).

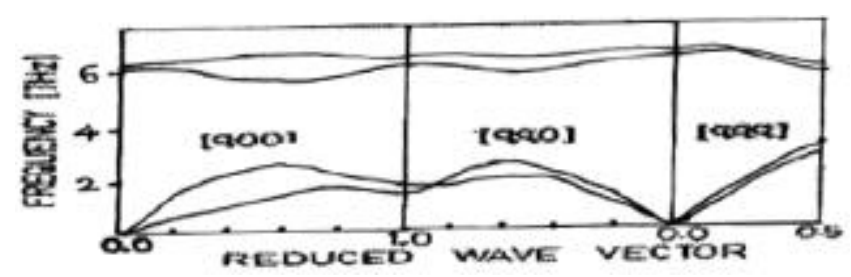

Figure 1. Phonon dispersion curve for USe.

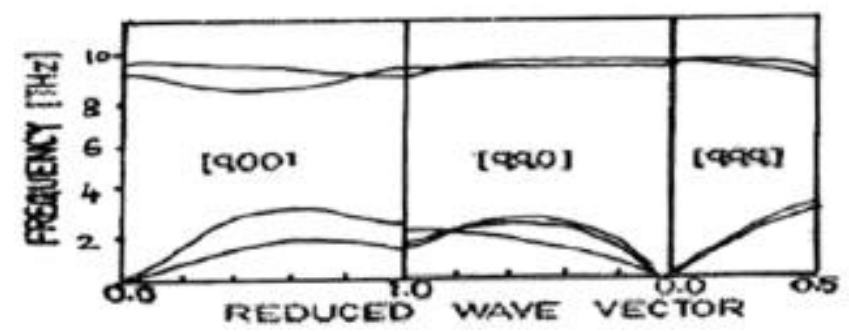

Figure 2. Phonon dispersion curve for US.

tion is the fact that the fermions are coupled to a lowenergy set of spin fluctuation. This may be compared with a value of $\mu^{*}=0.13$ in the case of metallic glass superconductors where the $T_{\mathrm{c}}$ value lies in the approximate range from 3-0.6 K (Altounian and Strom-Olsen 1983).

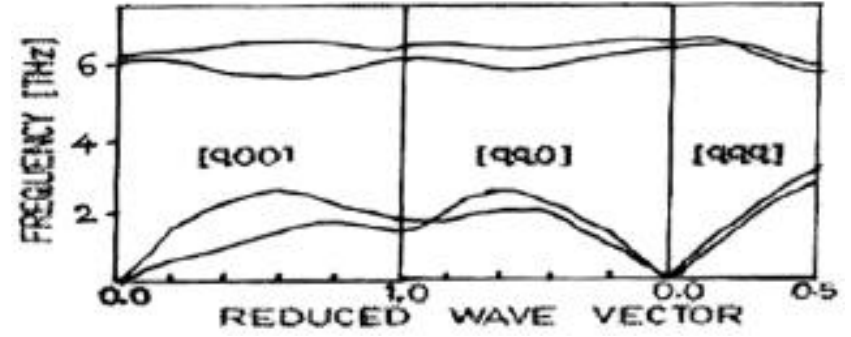

Figure 3. Phonon dispersion curve for UTe.

\section{Results and discussion}

Tables 1 and 2 are the input data and the model parameters for three-body force shell model. Tables 3-5 give the estimated values of phonon frequencies in the symmetry directions for US, USe and UTe. Figures 1-3 give the phonon dispersive curves for USe, US and UTe.

Some of the salient features are:

(i) The $L 0$ and $T 0$ frequencies in $q 00$ directions have a separation of 0.3561 and $0 \cdot 1273,0.24$ and 0.1067 and 0.0753 and $0.0568 \mathrm{THz}$ for US, USe and UTe, respectively. The $L A$ and $T A$ frequencies are separated by 0.0452 and 1.1786 , 0.0551 and 0.8920 and 0.0529 and $0.6540 \mathrm{THz}$, with a maximum separation. 
Table 6. $\quad \lambda_{\text {ee }}, \lambda_{\mathrm{sf}} \lambda_{\mathrm{D}}$ and $T_{\mathrm{c}}$ values.

\begin{tabular}{lccccc}
\hline & & & & \multicolumn{2}{c}{$T_{\mathrm{c}}$} \\
\cline { 5 - 6 } Compound & $\lambda_{\text {ee }}$ & $\lambda_{\text {sf }}$ & $\lambda_{\mathrm{D}}$ & $\mu^{*}=0.1$ & $\mu^{*}=0.12$ \\
\hline US & -0.06 & 0.33 & 138.71 & 0.212 & 0.103 \\
USe & -0.06 & 0.34 & 138.71 & 0.258 & 0.132 \\
UTe & -0.07 & 0.35 & 114.79 & 0.279 & 0.153 \\
\hline
\end{tabular}

(ii) For $q q 0$ direction the variation is small in optical mode for all compounds whereas the acoustical mode shows a wide variation.

(iii) For $q q q$ symmetry direction there appears to be an overlapping tendency between transverse and longitudinal frequencies in both optical and acoustical modes. The frequencies are almost identical for each direction. The trend is due to the fact that the compounds are more metallic than ionic.

(iv) Some of the earlier values are given for comparison in order to appreciate the theoretical approach.

In table $6, \lambda_{\text {ep }}, \lambda_{\mathrm{sf}}, \theta_{\mathrm{D}}$ and $T_{\mathrm{c}}$ values are presented. From the phonon frequencies obtained, the Debye temperatures have been estimated. Comparing these values with $210 \mathrm{~K}$ for $\mathrm{UPt}_{13}$ and $122 \mathrm{~K}$ for $\mathrm{UBe}_{13}$, the values estimated by us are reasonable. The $T_{\mathrm{c}}$ values obtained for $\mu^{*}=0 \cdot 1$ and $0 \cdot 12$ are given. The values down to $0 \mathrm{~K}$ region may be one day supported by experimental values.

\section{Conclusions}

The behaviour of $\mathrm{UPt}_{3}$ and $\mathrm{UBe}_{13}$ has been taken as the ground for this investigation and the lattice dynamical studies are used in predicting the phonon frequencies and also the superconducting temperature. The results provide scope for a new line of investigation.

\section{References}

Aeppli G, Bucher E and Shirave G 1985 Phys. Rev. B32 7579 Agarwal S K 1977 J. Phys. Chem. Solids 38199

Altounian Z and Strom-Olsen J O 1983 Phys. Rev. B27 4149

Dick B J and Overhauser A W 1958 Phys. Rev. 11290

Durai S, Venkatasubramanian N and Mohan S 1987 Curr. Sci. 56941

Jackman J A, Holden T M, Buyers W J L, DuPlessis P, De V, Vogt O and Genossar G 1986 Phys. Rev. B33 7144

Jha P K and Sanyal S P 1992 Phys. Rev. B46 3664

Kellermann E W 1940 Philos. Trans. R. Soc. (London) A238 513

McMillan W L 1968 Phys. Rev. 167331

Mohan S, Durai S and Vaidyanathan G 1986 Indian J. Phys. A60 137

Mohan S and Durai S 1988 Proc. Indian Natl. Sci. Acad. A54 920

Oguchi T and Freeman J 1985 Physica B135 468

Ott H R, Rudigier H, Rice T M, Veda K, Fisk Z and Smith J L 1984 Phys. Rev. Lett. 521915

Verma M P and Singh R K 1969 Phys. Status Solidi 33769

Verma M P and Agarwal S K 1973 Phys. Rev. B8 4880

Woods A D B, Cochran W and Brockhouse B N 1960 Phys. Rev. 119980 